\title{
Plant diversity, functional traits and soil conditions of grass savannas on lateritic crusts (bowé) in south eastern Burkina Faso
}

\begin{abstract}
Alexandra Zwarg, Marco Schmidt, Thomas Janßen, Karen Hahn, Georg Zizka
Summary: Grass savannas on lateritic crusts are characteristic landscape elements of the Sudanian savannas. In the W National Park and its surroundings in SE-Burkina Faso, plant diversity of savannas on and adjacent to bowé was assessed by a survey of 19 bowal areas with relevés along transects in each of these. The vegetation structure and species composition of the herb and shrub strata, soil depth, particle size and the concentration of $\mathrm{Na}^{+}, \mathrm{K}^{+}, \mathrm{Mg}^{2+}, \mathrm{Ca}^{2+}, \mathrm{H}^{+}, \mathrm{C}$ and $\mathrm{N}$ were recorded on each bowal and its surroundings. Our results show that soils on lateritic crusts are rather shallow and acidic compared to the surrounding savanna woodlands. Nutrient availability is hence comparatively low. The observed flora comprises 130 species mainly belonging to the families Combretaceae, Cyperaceae, Leguminosae and Poaceae with a predominance of therophytes and a comparatively high share of $\mathrm{C} 4$ species. In the pastures surrounding the National Park a higher species richness was found than inside the Park due to the occurrence of pioneers, ruderal and unpalatable plants. Savannas on lateritic crusts exhibit due to their extreme edaphic and hydrological conditions a specific flora distinct from their surroundings.
\end{abstract}

Key words: grass savanna, hardpan, phytodiversity, plant functional types, savanna woodland, Sudanian zone, West Africa

\section{Phytodiversité, tRAITS FONCTIONNELS ET CONDITIONS DU SOL DES SAVANES HERBEUSES SUR CUIRASSE LATÉRITIQUE (BOWÉ) AU SUD-EST DU BURKINA FASO}

Résumé: Les savanes herbeuses sur cuirasse lateritique sont des elements de paysage typiques de la savane soudanienne. Dans le Parc national de la W et ses environs au Sud-est du Burkina Faso, la phytodiversité des savanes sur bowé et à côté d'eux étais saisi sur 19 sites de bowal avec des relevés suivant des transects. La structure de la végétation et la composition spécifique des strates herbacée et ligneuse, la profondeur du sol, la taille du grain et la concentration de $\mathrm{Na}^{+}, \mathrm{K}^{+}, \mathrm{Mg}^{2+}, \mathrm{Ca}^{2+}$, $\mathrm{H}^{+}, \mathrm{C}$ et $\mathrm{N}$ étaient notés pour chaque bowal et ses environs. Nos résultants montrent que les sols sur cuirasse latéritique sont peu profonds et acidiques par rapport aux savanes voisines. La disponibilité de nutriments et relativement basse. Les 130 espèces observées pour la plupart font parti des familles Combretaceae, Cyperaceae, Leguminosae and Poaceae avec une prédominance des thérophytes et une proportion élevée des espèces C4. Dans les aires pâturées autour du parc national, une richesse spécifique plus élevée qu'à l'intérieur était trouvé par l'occurrence des espèces pionnières et des plantes rudéraux et non comestibles. Les savanes sur cuirasse latéritique montrent une flore distincte de ses environs à cause des extrêmes édaphiques et hydrologiques.

Mots clés: Afrique de l'Ouest, cuirasse latéritique, phytodiversité, savane boisée, savane herbeuse, types fonctionnels de plantes, zone soudanienne

\section{Phytodiversität, Lebensformen, Photosynthesewege und Bodenverhältnisse von Savannen Auf Laterit- KRUSTEN (BOWÉ) IM SÜDOSTEN VON BURKINA FASO}

Zusammenfassung: Grassavannen auf Lateritkrusten sind charakteristische Landschaftselemente der sudanischen Savannen. Im zu Burkina Faso gehörenden Teil des W-Nationalparks und seiner Umgebung wurde die pflanzliche Diversität der Savannen auf und in der Umgebung von 19 Bowé-Flächen entlang von Transekten untersucht. Vegetationsstruktur und Artenzusammensetzung der Kraut- und Gehölzschicht, Bodentiefe, Körnung und die Gehalte an $\mathrm{Na}^{+}, \mathrm{K}^{+}, \mathrm{Mg}^{2+}, \mathrm{Ca}^{2+}, \mathrm{H}^{+}, \mathrm{C}$ und N wurden auf jeder Fläche und in deren Umgebung ermittelt. Unsere Ergebnisse zeigen, dass die Böden auf den Lateritkrusten im Vergleich zu denen der umgebenden gehölzreichen Savannen ziemlich flach und sauer sind. Die Nährstoffverfügbarkeit ist daher vergleichsweise niedrig. Die Flora umfasst 130 Arten, insbesondere aus den Familien Combretaceae, Cyperaceae, Leguminosae und Poaceae, wobei Therophyten dominieren. Bemerkenswert ist auch ein verhältnismäßig hoher Anteil an C4 Arten. Auf den Nationalpark umgebenden beweideten Flächen wurde eine höhere Artenzahl gefunden als innerhalb des Parkes, was auf die höhere Zahl von Pionieren, ruderalen und ungenießbaren Arten zurückzuführen ist. Aufgrund ihrer extremen edaphischen und hydrologischen Bedingungen besitzen die Savannen auf den Lateritkrusten eine deutlich von den angrenzenden Flächen unterschiedene spezifische Vegetation.

Schlagworte: Bodenverhältnisse, Grassavannen, Lateritkrusten, Lebensform, Photosyntheseweg, Phytodiversität, Gehölzsavannen, Sudanzone, Westafrika

\section{INTRODUCTION}

Increasing land use and climate change are major drivers of the loss of biodiversity in tropical regions. Especially in West Africa due to population growth and resulting land use pressure, the natural flora and fauna is more and more restricted to wildlife reserves or non-arable habitats like e.g. mountain ridges, inselbergs and lateritic crusts (KöNIG et al. 2007). These habitats exhibit a rather uniform vegetation that differs from their surroundings (Fig.1) and often contain rare plant species, e.g. carnivores of the Lentibulariaceae and Droseraceae (MÜLLER 2007; POREMBSKI \& WATVE 2005).

In contrast to inselbergs and mountain ridges, only little information is available about habitat conditions and the vegetation cover of lateritic crusts as well as their importance for the savanna ecosystem of the Sudanian Zone. These 


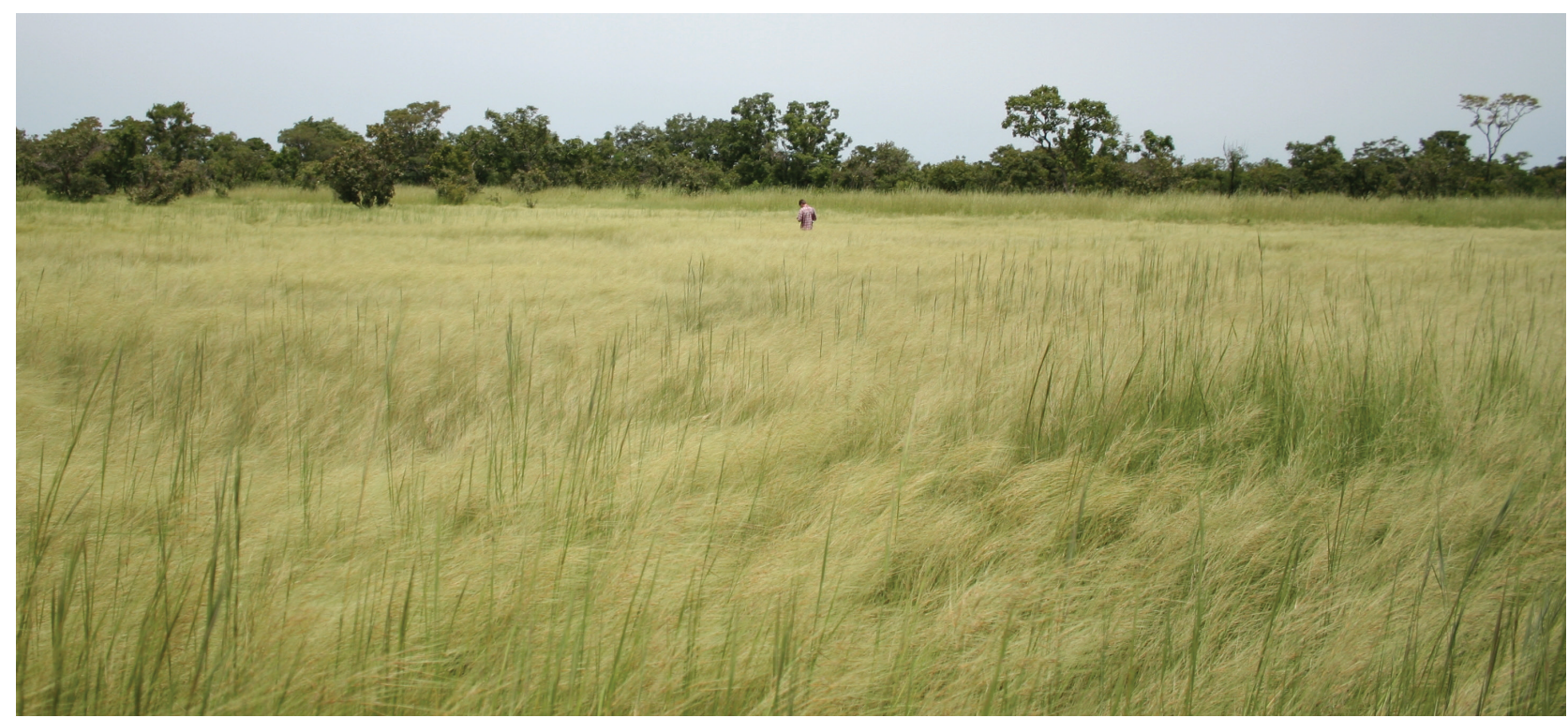

Fig. 1: A typical bowal in W National Park between Kabougou and Point Triple. The bowal is free of woody plants. / Un bowal typique dans le Parc National de la W entre Kabougou et Point Triple. Le bowal est dénué des plantes ligneuses. / Ein typischer Bowal im W-Nationalpark zwischen Kabougou und Point Triple. Der Bowal ist frei von Gehölzen.

landscape elements are typical for tropical semiarid areas where physical and chemical weathering is promoted and are hence widespread in West Africa. Their development takes place during humid periods when iron is leached from the top soils and transferred into deeper soil layers. Later, during dry periods, this material is hardened and becomes resistant to erosion (BROwN et al. 1994).

In West Africa, lateritic crusts form broad plains known there as bowé (sing.: bowal, pl.: bowé; derived from the Fula word stem woow-/boow- : monotony, uniformity; to get accustomed to something; pers. comm. Dr. Abdourahmane Diallo). The impermeable crusts are only covered by a thin soil layer. During the rainy season, they are often waterlogged and afterwards extremely dry. These harsh conditions restrain the development of a woody layer and lead to the formation of grass savannas, only occasionally interspersed by trees.

Bowé are usually not cultivated and pasturing intensity is comparatively low due to the low fodder quality of the dominating herbs. Mostly, they are visited by game during the rainy season (KROHMER 2004). Only a few general surveys on savanna vegetation in Burkina Faso, Mali and Benin (NASI 1994, HaHN 1996, KüpPeRs 1996, SieglstetTer 2002, KroHMER 2004) include descriptions of the species composition on lateritic crusts. Besides, a few studies on vegetation in temporarily wet habitats on lateritic crusts exist (MüLlER 2007). A complete and detailed survey of the phytodiversity and soil conditions of lateritic crusts as well as the role of human impact on these features does not exist.

\section{STUDY AREA}

The Study area is located in the Sudanian Zone of SE - Burkina Faso between $11^{\circ} 48^{\prime}$ and $12^{\circ} 12^{\prime} \mathrm{N}$ and $1^{\circ} 47^{\prime}$ and $2^{\circ} 24^{\prime}$ $\mathrm{E}$ within and adjacent to the $\mathrm{W}$ National Park (Fig. 2). This transnational reserve in Niger, Benin and Burkina Faso was founded in 1954 and belongs together with the Pendjari National Park, the Arly National Park and adjacent reser- ves and hunting zones to the so-called "WAP - complex". Since 1996, the park is listed as a UNESCO world heritage site. The semiarid climate in this region has a rainy season lasting from June to November and an average rainfall of $800 \mathrm{~mm} / \mathrm{a}$. The mean annual temperature is about $28^{\circ} \mathrm{C}$ (ASCENA 1993). The landscape is more or less flat except for solitary hills and the sandstone mountains of Gobnangou. On watersheds and lower slopes, albi-petric plinthosols, on upper and middle slopes, endosceleti-albic acrisols, and on lateritic crusts, shallow leptosols with low water-holding capacities can be found (FAO-ISRIC-ISSS 1998). The vegetation consists mainly of shrub and tree savannas with most species from the families of Combretaceae, Leguminosae and Poaceae. The fauna of the WAP-complex includes numerous large mammals, e.g. elephants, antelopes and buffalos, also endangered species such as leopards, cheetahs as well as many threatened birds (THIOLLAY 2006, LAMARQUE 2004, RABEIL 2003). The Southeast of Burkina Faso is mainly populated by the ethnic group of the Gulimanceba traditionally living on sorghum and maize farming; important minorities include the Djerma, Mossi and Fulani. A lot of wild plants are used by the local population. Characteristic for this area are the so-called park savannas that are formed by important useful trees like baobab (Adansonia digitata), shea butter tree (Vitellaria paradoxa) and African locust bean (Parkia biglobosa) left in the fields and fallows (BoFFA 1999). But also grazing and the regular setting of bushfires have their effects on vegetation and are key factors responsible for the genesis and maintenance of savannas (SANKARAN et al. 2008, GUINKo 1984).

\section{Methods}

\subsection{Site selection}

The selection of sites was based on ASTER-Satellite images from October 2006. In these images, the bowé can be easily distinguished from their surroundings by their sparse woody vegetation, resulting in a distinct reflection signal. Another 

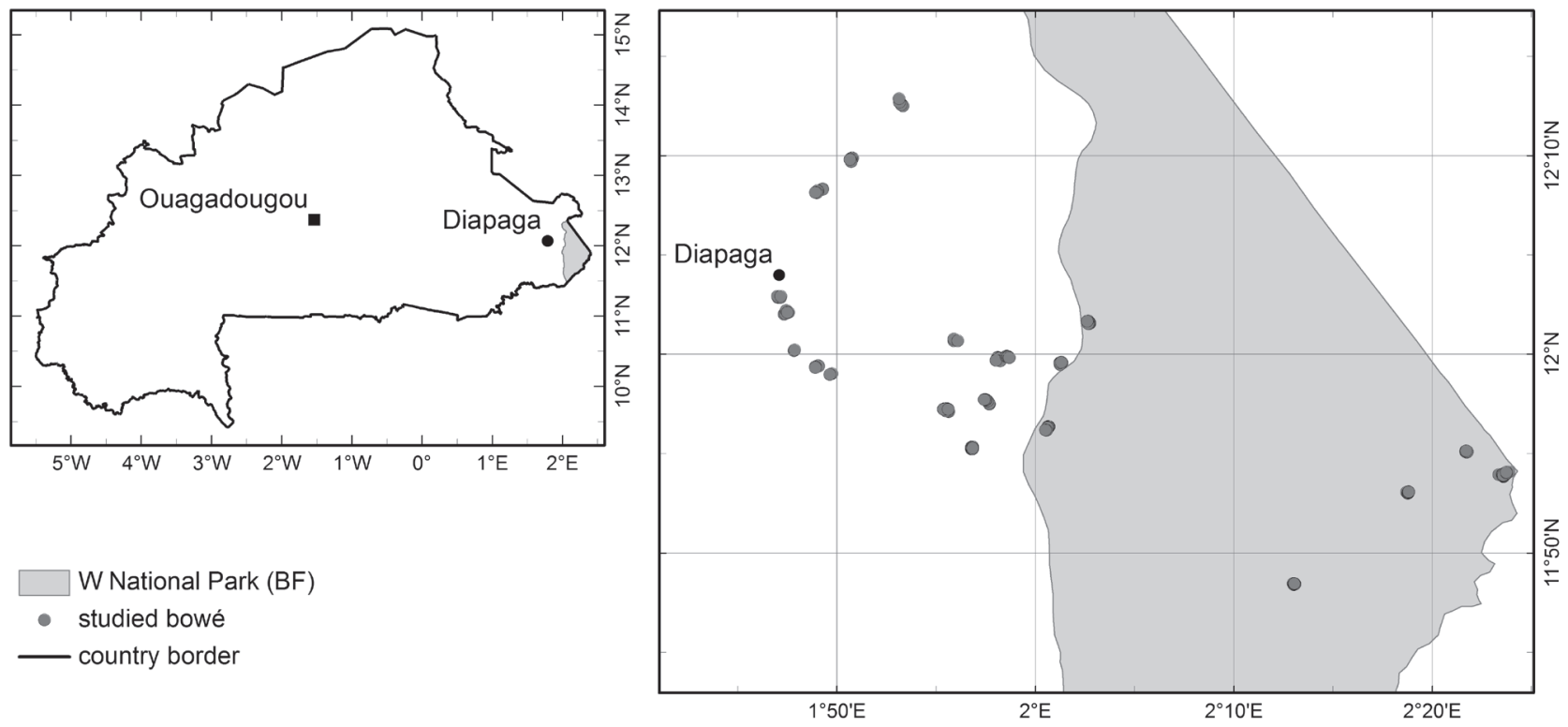

Fig. 2: Study area in the SE of Burkina Faso. Borders of W National Park are taken from IUCN \& UNEP-WCMC (2010). / Aire de recherché dans le Sud-est du Burkina Faso. La frontière du Parc National est prise d'IUCN \& UNEP-WCMC (2010). / Untersuchungsgebiet im Südosten Burkina Fasos. Grenzen des W-Nationalparks sind aus IUCN \& UNEP WCMC (2010).

criterion of choice was the accessibility of the sites, especially within the National Park where access was limited to the vicinity of roads. Surveys were conducted at the end of the rainy season from September to November in 2007 when many plants, e.g. most grasses were flowering and before the setting of fire started. On four sites in the protected area of W National Park as well as on 15 sites in several adjacent pastures soils, vegetation structure and floristic composition were analysed.

\subsection{Relevé plot design}

On each site relevé plots along three parallel transects were constructed. For the herb layer, a plot size of $10 \times 10 \mathrm{~m}^{2}$, and for the woody layer, a plot size of $30 \times 30 \mathrm{~m}^{2}$ were chosen. Soil samples were taken in each corner in $1 \mathrm{~m}$ distance from the edge and in the centre of the plot.

\subsection{Sampling of soil data}

In each plot soil depth was measured and five samples were taken from the topsoil and merged to a mixed sample. Sand fractions were separated by grading, silt and clay fractions by the pipette method (KöHN 1928). The pH-Value was measured by a replicate set of $\mathrm{pH}$ readings in 0.1 $\mathrm{N} \mathrm{KCl}$ according to Dewis \& Freitas (1970). For determination of cations $\left(\mathrm{Mg}^{2+}, \mathrm{Ca}^{2+}, \mathrm{K}^{+}, \mathrm{Na}^{+}\right)$, the method of MeHLich (1948) was used. Carbon and nitrogen were determined through dry combustion in oxygen by $\mathrm{CN}$-Analysers (LECO).

\subsection{Sampling of vegetation data}

Total coverage and mean height of vegetation as well as abundance of species of the herbaceous and ligneous stratum were recorded separately on each relevé plot. Species of the woody layer were separated at a height of $5 \mathrm{~m}$ in shrubs and trees. Cover was recorded in percentages. The complete re- levé data are stored in the West African Vegetation Database (JANSSEN et al. 2011, SCHMIDT et al. 2012).

Each species has been documented by herbarium specimens stored in the Herbarium Senckenbergianum (FR) of the Research Institute Senckenberg. The respective records are available via the Senckenberg collection database (http:// sesam.senckenberg.de/).

\subsection{Plant functional traits}

Information on life form and photosynthetic pathways was taken from the species list of the partial faunal reserve of Pama (MBAYNGONE et al. 2008) and the vegetation database VegDa (SCHMIDT 2006).

\subsection{Statistical Analysis}

Patterns of floristic compositional differences were detected by an ordination analysis in CANOCO (TER BRAAK 1988). Data were at first subjected to a detrended correspondence analysis (DCA) for obtaining the length of the floristic gradient. This was followed by a canonical correspondence analysis (CCA), conducted to test whether soil conditions or grazing account for the variance in species composition. A Monte Carlo permutation test was used to prove if the results of the ordination are significant (TER BRAAK 1988).

\section{Results}

\subsection{Soil}

Soils on the examined lateritic crusts are significantly less profound than in the surrounding savannas but show a great variety in depth because of cleavages in the crust or its uneven surface. Base saturations, $\mathrm{pH}$ values, $\mathrm{Ca}$ and $\mathrm{Mg}$ contents are considerably lower and the clay fraction is slightly higher on lateritic crusts than on the surroundings. 
Surprisingly humus and nitrogen show constantly high and $\mathrm{C} / \mathrm{N}$-ratio low values (Table 1 ).

\subsection{Species richness}

Within the 145 plots, 130 plant species were found. In the woody layer, 44 species are represented, 20 of them occurring on the bowé and 42 in the adjacent savannas. Of the 106 herb species, 84 occurred on lateritic crusts and 76 in the surrounding savanna. As compared to the sites within W National Park, more species were found in the surrounding pasture areas due to the occurrence of pioneers, ruderal and unpalatable plants. In general, the bowé are poor in species, on average only $12(+/-3)$ different species were found per plot which is, compared to other common vegetation types in this region, a very small number.

\subsection{Families}

In the woody layer, species from 19 families and in the herb layer from 26 families were found. Most of them belong to the Leguminosae and Combretaceae. In the herb layer the Poaceae are strongly represented. All grass species occur-

Table 1: Chemical and physical properties of the topsoils on lateritic crusts and their surrounding savannas. / Propriétés chimiques et physiques des horizons A sur cuirasses latéritiques et savanes avoisinants. / Chemische und physikalische Eigenschaften des Oberbodens auf Lateritkrusten und benachbarten Savannen.

\begin{tabular}{|c|c|c|c|c|c|c|c|c|c|c|c|c|c|}
\hline \multicolumn{14}{|c|}{ Soil parameters } \\
\hline Vegetation type & Depth * & $\mathrm{pH} * *$ & Base sat. & $\mathrm{CEC}$ & $\mathrm{Na}$ & K & $\mathrm{Mg} * *$ & $\mathrm{Ca}^{* *}$ & H-ions & Texture & $\mathrm{C}$ & $\mathrm{N}$ & $\mathrm{C} / \mathrm{N}$-ratio \\
\hline & $\mathrm{cm}$ & & $\%$ & \multicolumn{6}{|c|}{ mmol / ( $z^{*} 100 \mathrm{~g}$ Boden $)$} & clay:silt:sand & $\%$ & $\%$ & \\
\hline Bowé savanna & 10 & 5,4 & 51,4 & 13,3 & 0,17 & 0,25 & 1,36 & 5,12 & 6,37 & $16: 51: 33$ & 1,99 & 0,17 & 11,5 \\
\hline SD & $(5,8)$ & $(0,4)$ & $(14,1)$ & $(3,8)$ & $(0,26)$ & $(0,33)$ & $(0,7)$ & $(2,3)$ & $(2,45)$ & & $(0,71)$ & $(0,05)$ & $(1,5)$ \\
\hline $\begin{array}{l}\text { s urrounding } \\
\text { savanna }\end{array}$ & 20 & $\mathbf{5 , 9}$ & 68,5 & 15,1 & 0,09 & 0,33 & 2,08 & 8,44 & 4,18 & $11: 52: 37$ & 2,15 & 0,18 & 11,8 \\
\hline SD & (9) & $(0,5)$ & $(15,5)$ & $(7,8)$ & $(0,17)$ & $(0,31)$ & $(1,18)$ & $(6,16)$ & $(2,01)$ & & $(1,04)$ & $(0,09)$ & $(1,6)$ \\
\hline
\end{tabular}

ring in our plots are $\mathrm{C} 4$ plants (Table 2), known to have a high water and nitrogen use efficiency (e.g. Simioni et al. 2004). Another family, which is common in Burkina Faso, but in this study was only found on the bowe are the Cyperaceae. Other families that occur exclusively on the bowé plots (but are represented only by one or two species) are the Caryophyllaceae, Convallariaceae, Ophioglossaceae, Moraceae and Portulacaceae.

\subsection{Plant functional traits}

Differences were also found regarding the life form spectrum as well as photosynthetic pathways. On the bowé, the number of therophytes and $\mathrm{C} 4$ plants is higher than in the surrounding savanna which, in turn, contains considerably more phanerophytes and $\mathrm{C} 3$ plants. This can be ascribed to the adaptation of plants to the extremely short vegetation periods combined with shortage of water and nitrogen on lateritic crusts.

\subsection{Species composition}

The few woody species occurring on bowé are resistant against drought and have high regeneration potentials like e.g. Acacia macrostachya, Combretum glutinosum,
Combretum nigricans, Detarium microcarpum and Lannea microcarpa (Table 2). The herb layer is strongly dominated by the grasses Loudetia togoensis or Loudetiopsis kerstingii. Other common but not highly abundant species are Abildgaardia abortiva, Indigofera geminata and Polygala arenaria. Many of these species are mentioned by several authors (ATAHOLO 2001; LEBRUN et al. 1991; POILECOT 1995) as typical plants of bowé or other dry habitats with shallow soils. Some species occur that are known to be adapted to seasonal waterlogging, as e.g. the only fern Ophioglossum reticulatum, the rare Asparagaceae Eriospermum flagelliforme, Cyanotis lanata and Spermacoce filifolia.

In the surrounding savannas where water and soil conditions are more favourable, many more woody species occur, including Anogeissus leiocarpa, Combretum molle, Grewia lasiodiscus and Vitellaria paradoxa.

The herb layer is dominated by the grass Andropogon fastigiatus that is often accompanied by Andropogon gayanus and Tephrosia bracteolata. Another remarkable fact is, that some species do not occur on examined sites outside the reserve and vice versa. A species only found in the reserve is e.g. Ctenium elegans. Species found exclusively on the grazed plots are e.g. Waltheria indica, Hackelochloa granularis and Eragrostis turgida.

Table 2: List of species found on bowé (B) and adjacent savannas (S), alphabetically ordered by families with life forms (LF: C = chamaephyte, $T=$ therophyte, $\mathbf{P}=$ phanerophyte, $\mathbf{H P}=$ hemiparasite, $\mathrm{H}=$ hemicryptophyte), types of photosynthesis (PS), occurrence in herb (H) and woody layer $(\mathrm{W})$. / Liste d'espèces trouvées sur bowé $(\mathrm{B})$ et savanes avoisinants (S), en ordre alphabétique de familles avec types biologiques (LF: $\mathbf{C}=$ chaméphyte, $T=$ thérophyte, $P=$ phanérophyte, $H P=$ hémiparasite, $\mathbf{H}=$ hémikryptophyte), types de photosynthèse (PS), présence dans la strate herbacée $(\mathrm{H})$ ou ligneuse $(\mathrm{W})$. / Liste der auf Bowé $(B)$ und angrenzenden Savannen (S) gefundenen Arten, alphabetisch nach Familien geordnet, mit Lebensform $(\mathrm{LF}: \mathrm{C}=$ Chamaephyt, $\mathrm{T}=$ Therophyt, $\mathrm{P}=\mathrm{Phanerophyt}$, HP $=\mathbf{H e m i p a r a s i t}, \mathrm{H}=\mathrm{Hemikryp}-$ tophyt), Photosynthesetyp (PS) und Vorkommen in Kraut- (H) oder Gehölzschicht (W).

\begin{tabular}{|l|l|l|l|l|l|}
\hline Species & LF & PS & H & W & B \\
\hline Acanthaceae & & & & \\
\hline Blepharis maderaspatensis Heyne ex Roth & T & C3 & x & x \\
\hline Justicia insularis T. Anderson & P & C3 & $\mathrm{x}$ & $\mathrm{x}$ \\
\hline
\end{tabular}




\begin{tabular}{|c|c|c|c|c|c|c|}
\hline Species & LF & PS & $\mathbf{H}$ & W & B & $\mathbf{S}$ \\
\hline Lepidagathis anobrya Nees & $\mathrm{P}$ & $\mathrm{C} 3$ & $\mathrm{x}$ & & $\mathrm{x}$ & $\mathrm{x}$ \\
\hline Monechma ciliatum (Jacq.) Milne-Redh. & $\mathrm{T}$ & $\mathrm{C} 3$ & $\mathrm{x}$ & & $\mathrm{x}$ & $\mathrm{x}$ \\
\hline \multicolumn{7}{|l|}{ Amaranthaceae } \\
\hline Achyranthes aspera $\mathrm{L}$. & $\mathrm{T}$ & $\mathrm{C} 3$ & $\mathrm{x}$ & & $\mathrm{x}$ & $\mathrm{x}$ \\
\hline Pandiaka angustifolia (Vahl) Hepper & $\mathrm{T}$ & $\mathrm{C} 3$ & $\mathrm{x}$ & & $\mathrm{x}$ & $\mathrm{x}$ \\
\hline \multicolumn{7}{|l|}{ Anacardiaceae } \\
\hline Lannea acida A. Rich. & $\mathrm{P}$ & $\mathrm{C} 3$ & & $\mathrm{x}$ & $\mathrm{x}$ & $\mathrm{x}$ \\
\hline Lannea microcarpa Engl. \& K. Krause & $\mathrm{P}$ & $\mathrm{C} 3$ & & $\mathrm{x}$ & $\mathrm{x}$ & $\mathrm{x}$ \\
\hline \multicolumn{7}{|l|}{ Annonaceae } \\
\hline Annona senegalensis Pers. & $\mathrm{P}$ & $\mathrm{C} 3$ & $\mathrm{x}$ & $\mathrm{x}$ & $\mathrm{x}$ & $\mathrm{x}$ \\
\hline \multicolumn{7}{|l|}{ Asparagaceae } \\
\hline Chlorophytum laxum $\mathrm{R}$. Br. & G & $\mathrm{C} 3$ & $\mathrm{x}$ & & 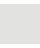 & $\mathrm{x}$ \\
\hline Eriospermum flagelliforme Baker & G & $\mathrm{C} 3$ & $\mathrm{x}$ & & $\mathrm{x}$ & \\
\hline \multicolumn{7}{|l|}{ Asteraceae } \\
\hline Aspilia bussei O. Hoffm. \& Muschl. & $\mathrm{T}$ & $\mathrm{C} 3$ & $\mathrm{x}$ & & $\mathrm{x}$ & $\mathrm{x}$ \\
\hline Aspilia paludosa Berhaut & $\mathrm{T}$ & $\mathrm{C} 3$ & $\mathrm{x}$ & & & $\mathrm{x}$ \\
\hline \multicolumn{7}{|l|}{ Bignoniaceae } \\
\hline Stereospermum kunthianum Cham. & $\mathrm{P}$ & $\mathrm{C} 3$ & $\mathrm{x}$ & & 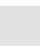 & $\mathrm{x}$ \\
\hline \multicolumn{7}{|l|}{ Bixaceae } \\
\hline Cochlospermum planchonii Hook. f. & $\mathrm{C}$ & $\mathrm{C} 3$ & $\mathrm{x}$ & & & $\mathrm{x}$ \\
\hline \multicolumn{7}{|l|}{ Caryophyllaceae } \\
\hline Polycarpaea corymbosa (L.) Lam. & $\mathrm{T}$ & $\mathrm{C} 4$ & $\mathrm{x}$ & & $\mathrm{x}$ & \\
\hline \multicolumn{7}{|l|}{ Celastraceae } \\
\hline Gymnosporia senegalensis (Lam.) Loes. & $\mathrm{P}$ & $\mathrm{C} 3$ & & $\mathrm{x}$ & & $\mathrm{x}$ \\
\hline \multicolumn{7}{|l|}{ Combretaceae } \\
\hline Anogeissus leiocarpa (DC.) Guill. \& Perr. & $\mathrm{P}$ & $\mathrm{C} 3$ & & $\mathrm{x}$ & & $\mathrm{x}$ \\
\hline Combretum collinum Fresen. & $\mathrm{P}$ & $\mathrm{C} 3$ & $\mathrm{x}$ & $\mathrm{x}$ & $\mathrm{x}$ & $\mathrm{x}$ \\
\hline Combretum glutinosum Perr. ex DC. & $\mathrm{P}$ & $\mathrm{C} 3$ & $\mathrm{x}$ & $\mathrm{x}$ & $\mathrm{x}$ & $\mathrm{x}$ \\
\hline Combretum micranthum G. Don & $\mathrm{P}$ & $\mathrm{C} 3$ & & $\mathrm{x}$ & & $\mathrm{x}$ \\
\hline Combretum molle R. Br. ex G. Don & $\mathrm{P}$ & $\mathrm{C} 3$ & $\mathrm{x}$ & $\mathrm{x}$ & & $\mathrm{x}$ \\
\hline Combretum nigricans Lepr. ex Guill. \& Perr. & $\mathrm{P}$ & $\mathrm{C} 3$ & $\mathrm{x}$ & $\mathrm{x}$ & $\mathrm{x}$ & $\mathrm{x}$ \\
\hline Guiera senegalensis J.F. Gmel. & $\mathrm{P}$ & $\mathrm{C} 3$ & $\mathrm{x}$ & $\mathrm{x}$ & & $\mathrm{x}$ \\
\hline Pteleopsis suberosa Engl. \& Diels & $\mathrm{P}$ & $\mathrm{C} 3$ & $\mathrm{x}$ & $\mathrm{x}$ & $\mathrm{x}$ & $\mathrm{x}$ \\
\hline Terminalia macroptera Guill. \& Perr. & $\mathrm{P}$ & $\mathrm{C} 3$ & & $\mathrm{x}$ & & $\mathrm{x}$ \\
\hline \multicolumn{7}{|l|}{ Commelinaceae } \\
\hline Commelina nigritana var. gambiae Benth. & $\mathrm{C}$ & $\mathrm{C} 3$ & $\mathrm{x}$ & & $\mathrm{x}$ & \\
\hline Cyanotis lanata Benth. & $\mathrm{T}$ & $\mathrm{C} 3$ & $\mathrm{x}$ & & $\mathrm{x}$ & $\mathrm{x}$ \\
\hline \multicolumn{7}{|l|}{ Convolvulaceae } \\
\hline Ipomoea coscinosperma Hochst. ex Choisy & $\mathrm{T}$ & $\mathrm{C} 3$ & $\mathrm{x}$ & & $\mathrm{x}$ & $\mathrm{x}$ \\
\hline Ipomoea eriocarpa $\mathrm{R}$. Br. & $\mathrm{T}$ & $\mathrm{C} 3$ & $\mathrm{x}$ & & $\mathrm{x}$ & $\mathrm{x}$ \\
\hline \multicolumn{7}{|l|}{ Cyperaceae } \\
\hline Abildgaardia abortiva (Steud.) Lye & $\mathrm{T}$ & $\mathrm{C} 3$ & $\mathrm{x}$ & & $\mathrm{x}$ & \\
\hline Cyperus reduncus Hochst. ex Boeck. & $\mathrm{T}$ & $\mathrm{C} 3$ & $\mathrm{x}$ & & $\mathrm{x}$ & \\
\hline Kyllinga erecta Schumach. & G & $\mathrm{C} 4$ & $\mathrm{x}$ & & $\mathrm{x}$ & \\
\hline Mariscus squarrosus (L.) C.B. Cl. & $\mathrm{T}$ & $\mathrm{C} 4$ & $\mathrm{x}$ & & $\mathrm{x}$ & \\
\hline Pycreus pumilus (L.) Domin. & $\mathrm{T}$ & $\mathrm{C} 4$ & $\mathrm{x}$ & & $\mathrm{x}$ & \\
\hline Lipocarpha kernii Raymond & $\mathrm{T}$ & $\mathrm{C} 4$ & $\mathrm{x}$ & & $\mathrm{x}$ & \\
\hline Scleria sphaerocarpa (E.A.Rob.) Napper & $\mathrm{T}$ & $\mathrm{C} 3$ & $\mathrm{x}$ & & $\mathrm{x}$ & \\
\hline \multicolumn{7}{|l|}{ Euphorbiaceae } \\
\hline Euphorbia convolvuloides Hochst. ex Benth. & $\mathrm{T}$ & $\mathrm{C} 3$ & $\mathrm{x}$ & & $\mathrm{x}$ & \\
\hline \multicolumn{7}{|l|}{ Fabaceae, subfam. Caesalpinoideae } \\
\hline Burkea africana Hook. & $\mathrm{P}$ & $\mathrm{C} 3$ & $\mathrm{x}$ & $\mathrm{x}$ & & $\mathrm{x}$ \\
\hline Cassia absus L. & $\mathrm{C}$ & $\mathrm{C} 3$ & $\mathrm{x}$ & & $\mathrm{x}$ & \\
\hline Cassia mimosoides $\mathrm{L}$. & $\mathrm{T}$ & $\mathrm{C} 3$ & $\mathrm{x}$ & & $\mathrm{x}$ & $\mathrm{x}$ \\
\hline Cassia sieberiana DC. & $\mathrm{P}$ & $\mathrm{C} 3$ & $\mathrm{x}$ & $\mathrm{x}$ & $\mathrm{x}$ & $\mathrm{x}$ \\
\hline Detarium microcarpum Guill. \& Perr. & $\mathrm{P}$ & $\mathrm{C} 3$ & $\mathrm{x}$ & $\mathrm{x}$ & $\mathrm{x}$ & $\mathrm{x}$ \\
\hline Isoberlinia doka Craib \& Stapf & $\mathrm{P}$ & $\mathrm{C} 3$ & & $\mathrm{x}$ & & $\mathrm{x}$ \\
\hline Piliostigma thonningii (Schumach.) Milne-Redh. & $\mathrm{P}$ & $\mathrm{C} 3$ & & $\mathrm{x}$ & & $\mathrm{x}$ \\
\hline Tamarindus indica $\mathrm{L}$. & $\mathrm{P}$ & $\mathrm{C} 3$ & & $\mathrm{x}$ & & $\mathrm{X}$ \\
\hline
\end{tabular}




\begin{tabular}{|c|c|c|c|c|c|c|}
\hline Species & $\mathbf{L F}$ & PS & $\mathbf{H}$ & W & B & $\mathbf{S}$ \\
\hline \multicolumn{7}{|l|}{ Fabaceae, subfam. Mimosoideae } \\
\hline Acacia dudgeonii Craib & $\mathrm{P}$ & $\mathrm{C} 3$ & & $\mathrm{x}$ & & $\mathrm{x}$ \\
\hline Acacia hockii De Wild. & $\mathrm{P}$ & $\mathrm{C} 3$ & & $\mathrm{x}$ & & $\mathrm{x}$ \\
\hline Acacia macrostachya Reichb. ex G. Don & $\mathrm{P}$ & $\mathrm{C} 3$ & $\mathrm{x}$ & $\mathrm{x}$ & $\mathrm{x}$ & $\mathrm{x}$ \\
\hline Albizia chevalieri Harms & $\mathrm{P}$ & $\mathrm{C} 3$ & & $\mathrm{x}$ & $\mathrm{x}$ & \\
\hline Dichrostachys cinerea (L.) Wright \& Arn. & $\mathrm{P}$ & $\mathrm{C} 3$ & $\mathrm{x}$ & $\mathrm{x}$ & & $\mathrm{x}$ \\
\hline Entada africana Guill. \& Perr. & $\mathrm{P}$ & $\mathrm{C} 3$ & $\mathrm{x}$ & $\mathrm{x}$ & & $\mathrm{x}$ \\
\hline Prosopis africana (Guill. \& Perr.) Taub. & $\mathrm{P}$ & $\mathrm{C} 3$ & & $\mathrm{x}$ & $\mathrm{x}$ & $\mathrm{x}$ \\
\hline \multicolumn{7}{|l|}{ Fabaceae, subfam. Papilionoideae } \\
\hline Alysicarpus ovalifolius (Schum. \& Thonn.) J. Léonard & $\mathrm{T}$ & $\mathrm{C} 3$ & $\mathrm{x}$ & & $\mathrm{x}$ & \\
\hline Crotalaria goreensis Guill. \& Perr. & $\mathrm{P}$ & $\mathrm{C} 3$ & $\mathrm{x}$ & & & $\mathrm{x}$ \\
\hline Crotalaria leprieurii Guill. \& Perr. & $\mathrm{T}$ & $\mathrm{C} 3$ & $\mathrm{x}$ & & $\mathrm{x}$ & $\mathrm{x}$ \\
\hline Desmodium adscendens (Sw.) DC. & $\mathrm{C}$ & $\mathrm{C} 3$ & $\mathrm{x}$ & & $\mathrm{x}$ & \\
\hline Desmodium velutinum (Willd.) DC. & $\mathrm{C}$ & $\mathrm{C} 3$ & $\mathrm{x}$ & & & $\mathrm{x}$ \\
\hline Indigofera bracteolata DC. & $\mathrm{C}$ & $\mathrm{C} 3$ & $\mathrm{x}$ & & $\mathrm{x}$ & $\mathrm{x}$ \\
\hline Indigofera congolensis De Wild. \& T. Durand & $\mathrm{T}$ & $\mathrm{C} 3$ & $\mathrm{x}$ & & $\mathrm{x}$ & \\
\hline Indigofera dendroides Jacq. & $\mathrm{T}$ & $\mathrm{C} 3$ & $\mathrm{x}$ & & $\mathrm{x}$ & $\mathrm{x}$ \\
\hline Indigofera geminata Baker & $\mathrm{T}$ & $\mathrm{C} 3$ & $\mathrm{x}$ & & $\mathrm{x}$ & \\
\hline Indigofera senegalensis Lam. & $\mathrm{T}$ & $\mathrm{C} 3$ & $\mathrm{x}$ & & $\mathrm{x}$ & \\
\hline Indigofera stenophylla Guill. \& Perr. & $\mathrm{T}$ & $\mathrm{C} 3$ & $\mathrm{x}$ & & $\mathrm{x}$ & \\
\hline Melliniella micrantha Harms & $\mathrm{T}$ & $\mathrm{C} 3$ & $\mathrm{x}$ & & $\mathrm{x}$ & $\mathrm{x}$ \\
\hline Pterocarpus erinaceus Poir. & $\mathrm{P}$ & $\mathrm{C} 3$ & $\mathrm{x}$ & $\mathrm{x}$ & $\mathrm{x}$ & $\mathrm{x}$ \\
\hline Stylosanthes erecta P. Beauv. & $\mathrm{C}$ & $\mathrm{C} 3$ & $\mathrm{x}$ & & & $\mathrm{x}$ \\
\hline Tephrosia bracteolata Guill. \& Perr. & $\mathrm{T}$ & $\mathrm{C} 3$ & $\mathrm{x}$ & & $\mathrm{x}$ & $\mathrm{x}$ \\
\hline Tephrosia pedicellata Baker & $\mathrm{T}$ & $\mathrm{C} 3$ & $\mathrm{x}$ & & $\mathrm{x}$ & $\mathrm{x}$ \\
\hline Tephrosia platycarpa Guill. \& Perr. & $\mathrm{T}$ & $\mathrm{C} 3$ & $\mathrm{x}$ & & $\mathrm{x}$ & $\mathrm{x}$ \\
\hline Xeroderris stuhlmannii (Taub.) Mendonca \& E.P. Sousa & $\mathrm{P}$ & $\mathrm{C} 3$ & & $\mathrm{x}$ & & $\mathrm{x}$ \\
\hline Zornia glochidiata Reichb. ex DC. & $\mathrm{T}$ & $\mathrm{C} 3$ & $\mathrm{x}$ & & $\mathrm{x}$ & \\
\hline \multicolumn{7}{|l|}{ Lamiaceae } \\
\hline Hyptis spicigera Lam. & $\mathrm{T}$ & $\mathrm{C} 3$ & $\mathrm{x}$ & & $\mathrm{x}$ & $\mathrm{x}$ \\
\hline Platostoma africanum P. Beauv. & $\mathrm{T}$ & $\mathrm{C} 3$ & $\mathrm{x}$ & & & $\mathrm{x}$ \\
\hline Tinnea barteri Gürke & $\mathrm{C}$ & $\mathrm{C} 3$ & $\mathrm{x}$ & & & $\mathrm{x}$ \\
\hline Vitex simplicifolia Oliv. & $\mathrm{P}$ & $\mathrm{C} 3$ & & $\mathrm{x}$ & & $\mathrm{x}$ \\
\hline \multicolumn{7}{|l|}{ Loganiaceae } \\
\hline Strychnos spinosa Lam. & $\mathrm{P}$ & $\mathrm{C} 3$ & & $\mathrm{x}$ & $\mathrm{x}$ & $\mathrm{x}$ \\
\hline \multicolumn{7}{|l|}{ Malvaceae } \\
\hline Bombax costatum Pellegr. \& Vuillet & $\mathrm{P}$ & $\mathrm{C} 3$ & $\mathrm{x}$ & $\mathrm{x}$ & & $\mathrm{x}$ \\
\hline Corchorus tridens L. & $\mathrm{T}$ & $\mathrm{C} 3$ & $\mathrm{x}$ & & $\mathrm{x}$ & \\
\hline Grewia cissoides Hutch. \& Dalziel & $\mathrm{C}$ & $\mathrm{C} 3$ & $\mathrm{x}$ & & & $\mathrm{x}$ \\
\hline Grewia lasiodiscus K. Schum. & $\mathrm{P}$ & $\mathrm{C} 3$ & $\mathrm{x}$ & $\mathrm{x}$ & $\mathrm{x}$ & $\mathrm{x}$ \\
\hline Hibiscus cannabinus L. & $\mathrm{T}$ & $\mathrm{C} 3$ & $\mathrm{x}$ & & $\mathrm{x}$ & $\mathrm{x}$ \\
\hline Sida alba $\mathrm{L}$ & $\mathrm{T}$ & $\mathrm{C} 3$ & $\mathrm{x}$ & & $\mathrm{x}$ & $\mathrm{x}$ \\
\hline Sterculia setigera Delile & $\mathrm{P}$ & $\mathrm{C} 3$ & $\mathrm{x}$ & $\mathrm{x}$ & & $\mathrm{x}$ \\
\hline Triumfetta rhomboidea Jacq. & $\mathrm{C}$ & $\mathrm{C} 3$ & $\mathrm{x}$ & & $\mathrm{x}$ & $\mathrm{x}$ \\
\hline Waltheria indica $\mathrm{L}$. & $\mathrm{C}$ & $\mathrm{C} 3$ & $\mathrm{x}$ & & $\mathrm{x}$ & $\mathrm{x}$ \\
\hline Wissadula rostrata (Schumach.) Hook.f. & $\mathrm{T}$ & $\mathrm{C} 3$ & $\mathrm{x}$ & & $\mathrm{x}$ & $\mathrm{x}$ \\
\hline \multicolumn{7}{|l|}{ Meliaceae } \\
\hline Khaya senegalensis (Desr.) A. Juss. & $\mathrm{P}$ & $\mathrm{C} 3$ & & $\mathrm{x}$ & 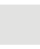 & $\mathrm{x}$ \\
\hline \multicolumn{7}{|l|}{ Moraceae } \\
\hline Ficus glumosa Delile & $\mathrm{P}$ & $\mathrm{C} 3$ & & $\mathrm{x}$ & $\mathrm{x}$ & \\
\hline \multicolumn{7}{|l|}{ Olacaceae } \\
\hline Ximenia americana $\mathrm{L}$. & $\mathrm{P}$ & $\mathrm{C} 3$ & & $\mathrm{x}$ & & $\mathrm{x}$ \\
\hline \multicolumn{7}{|l|}{ Ophioglossaceae } \\
\hline Ophioglossum reticulatum $\mathrm{L}$. & G & $\mathrm{C} 3$ & $\mathrm{x}$ & & $\mathrm{x}$ & \\
\hline \multicolumn{7}{|l|}{ Orobanchaceae } \\
\hline Buchnera hispida Buch.-Ham. ex D. Don & HP & $\mathrm{C} 3$ & $\mathrm{x}$ & & $\mathrm{x}$ & $\mathrm{x}$ \\
\hline Striga asiatica Kuntze & HP & C3 & $\mathrm{x}$ & & $\mathrm{x}$ & \\
\hline Striga aspera (Willd.) Benth. & HP & $\mathrm{C} 3$ & $\mathrm{x}$ & & $\mathrm{x}$ & $\mathrm{x}$ \\
\hline \multicolumn{7}{|l|}{ Phyllanthaceae } \\
\hline Bridelia scleroneura Muell.Arg. & $\mathrm{P}$ & $\mathrm{C} 3$ & $\mathrm{x}$ & $\mathrm{x}$ & $\mathrm{x}$ & $\mathrm{x}$ \\
\hline
\end{tabular}




\begin{tabular}{|c|c|c|c|c|c|c|}
\hline Species & LF & PS & $\mathbf{H}$ & W & B & $\mathbf{S}$ \\
\hline Flueggea virosa (Roxb. ex Willd.) Voigt & $\mathrm{P}$ & $\mathrm{C} 3$ & $\mathrm{x}$ & $\mathrm{x}$ & & $\mathrm{x}$ \\
\hline Phyllanthus amarus Schum. \& Thonn. & $\mathrm{P}$ & $\mathrm{C} 3$ & $\mathrm{x}$ & & $\mathrm{x}$ & \\
\hline \multicolumn{7}{|l|}{ Poaceae } \\
\hline Andropogon fastigiatus Sw. & $\mathrm{T}$ & $\mathrm{C} 4$ & $\mathrm{x}$ & & $\mathrm{x}$ & $\mathrm{x}$ \\
\hline Andropogon gayanus Kunth & $\mathrm{H}$ & $\mathrm{C} 4$ & $\mathrm{x}$ & & & $\mathrm{x}$ \\
\hline Aristida kerstingii Pilg. & $\mathrm{T}$ & $\mathrm{C} 4$ & $\mathrm{x}$ & & $\mathrm{x}$ & \\
\hline Brachiaria villosa (Lam.) A. Camus & $\mathrm{T}$ & $\mathrm{C} 4$ & $\mathrm{x}$ & & $\mathrm{x}$ & $\mathrm{x}$ \\
\hline Ctenium elegans Kunth & $\mathrm{T}$ & $\mathrm{C} 4$ & $\mathrm{x}$ & & $\mathrm{x}$ & \\
\hline Digitaria gayana (Kunth) Stapf ex A. Chev. & $\mathrm{T}$ & $\mathrm{C} 4$ & $\mathrm{x}$ & & $\mathrm{x}$ & $\mathrm{x}$ \\
\hline Eragrostis tremula Hochst. ex Steud. & $\mathrm{T}$ & $\mathrm{C} 4$ & $\mathrm{x}$ & & & $\mathrm{x}$ \\
\hline Eragrostis turgida (Schumach.) De Wild. & $\mathrm{T}$ & $\mathrm{C} 4$ & $\mathrm{x}$ & & $\mathrm{x}$ & \\
\hline Hackelochloa granularis (L.) Kuntze & $\mathrm{T}$ & $\mathrm{C} 4$ & $\mathrm{x}$ & & $\mathrm{x}$ & $\mathrm{x}$ \\
\hline Loudetia flavida (Stapf) C.E. Hubb. & $\mathrm{H}$ & $\mathrm{C} 4$ & $\mathrm{x}$ & & $\mathrm{x}$ & $\mathrm{x}$ \\
\hline Loudetia togoensis (Pilg.) C.E. Hubb. & $\mathrm{T}$ & $\mathrm{C} 4$ & $\mathrm{x}$ & & $\mathrm{x}$ & $\mathrm{x}$ \\
\hline Loudetiopsis kerstingii (Pilg.) Conert & $\mathrm{T}$ & $\mathrm{C} 4$ & $\mathrm{x}$ & & $\mathrm{x}$ & $\mathrm{x}$ \\
\hline Microchloa indica (L. f.) P. Beauv. & $\mathrm{T}$ & $\mathrm{C} 4$ & $\mathrm{x}$ & & $\mathrm{x}$ & $\mathrm{x}$ \\
\hline Panicum pansum Rendle & $\mathrm{T}$ & $\mathrm{C} 4$ & $\mathrm{x}$ & & $\mathrm{x}$ & $\mathrm{x}$ \\
\hline Pennisetum pedicellatum Trin. & $\mathrm{T}$ & $\mathrm{C} 4$ & $\mathrm{x}$ & & $\mathrm{x}$ & $\mathrm{x}$ \\
\hline Pennisetum polystachion (L.) Schult. & $\mathrm{T}$ & $\mathrm{C} 4$ & $\mathrm{x}$ & & $\mathrm{x}$ & $\mathrm{x}$ \\
\hline Schoenefeldia gracilis Kunth & $\mathrm{T}$ & $\mathrm{C} 4$ & $\mathrm{x}$ & & & $\mathrm{x}$ \\
\hline Setaria pumila (Poir.) Roem. \& Schult. & $\mathrm{T}$ & $\mathrm{C} 4$ & $\mathrm{x}$ & & $\mathrm{x}$ & $\mathrm{x}$ \\
\hline Sporobolus festivus Hochst. ex A. Rich. & $\mathrm{H}$ & $\mathrm{C} 4$ & $\mathrm{x}$ & & $\mathrm{x}$ & $\mathrm{x}$ \\
\hline Tripogon minimus (A. Rich.) Hochst. ex Steud. & $\mathrm{H}$ & $\mathrm{C} 4$ & $\mathrm{x}$ & & $\mathrm{x}$ & $\mathrm{x}$ \\
\hline \multicolumn{7}{|l|}{ Polygalaceae } \\
\hline Polygala arenaria Willd. & $\mathrm{T}$ & $\mathrm{C} 3$ & $\mathrm{x}$ & & $\mathrm{x}$ & \\
\hline Polygala multiflora Poir. & $\mathrm{T}$ & $\mathrm{C} 3$ & $\mathrm{x}$ & & $\mathrm{x}$ & $\mathrm{x}$ \\
\hline Securidaca longipedunculata Fresen. & $\mathrm{P}$ & $\mathrm{C} 3$ & & $\mathrm{x}$ & & $\mathrm{x}$ \\
\hline \multicolumn{7}{|l|}{ Portulacaceae } \\
\hline Portulaca foliosa Ker Gawl. & $\mathrm{C}$ & $\mathrm{C} 3$ & $\mathrm{x}$ & & $\mathrm{x}$ & \\
\hline \multicolumn{7}{|l|}{ Rhamnaceae } \\
\hline Ziziphus abyssinica Hochst. ex A. Rich. & $P$ & $\mathrm{C} 3$ & & $\mathrm{x}$ & & $\mathrm{x}$ \\
\hline \multicolumn{7}{|l|}{ Rubiaceae } \\
\hline Crossopteryx febrifuga (Afzel. ex G. Don) Benth. & $\mathrm{P}$ & $\mathrm{C} 3$ & $\mathrm{x}$ & $\mathrm{x}$ & $\mathrm{x}$ & $\mathrm{x}$ \\
\hline Feretia apodanthera Delile & $\mathrm{P}$ & $\mathrm{C} 3$ & & $\mathrm{x}$ & & $\mathrm{x}$ \\
\hline Kohautia tenuis (Bowdich) Mabb. & $\mathrm{T}$ & $\mathrm{C} 3$ & $\mathrm{x}$ & & $\mathrm{x}$ & \\
\hline Mitracarpus hirtus (L.) DC. & $\mathrm{T}$ & $\mathrm{C} 3$ & $\mathrm{x}$ & & $\mathrm{x}$ & \\
\hline Spermacoce filifolia (Schumach. \& Thonn.) J.P.Lebrun \& Stork & $\mathrm{T}$ & $\mathrm{C} 3$ & $\mathrm{x}$ & & $\mathrm{x}$ & $\mathrm{x}$ \\
\hline Spermacoce stachydea DC. & $\mathrm{T}$ & $\mathrm{C} 3$ & $\mathrm{x}$ & & $\mathrm{x}$ & $\mathrm{x}$ \\
\hline \multicolumn{7}{|l|}{ Sapotaceae } \\
\hline Vitellaria paradoxa C.F. Gaertn. & $\mathrm{P}$ & $\mathrm{C} 3$ & & $\mathrm{x}$ & $\mathrm{x}$ & $\mathrm{x}$ \\
\hline \multicolumn{7}{|l|}{ Zygophyllaceae } \\
\hline Balanites aegyptiaca Delile & $\mathrm{P}$ & $\mathrm{C} 3$ & & $\mathrm{x}$ & $\mathrm{x}$ & $x$ \\
\hline
\end{tabular}

\subsection{Ordination}

The DCA axis 1 showed a gradient length of 4.1 which means that species are unimodally distributed.

In the CCA, the significant effect of soil conditions on compositional change was the variable most strongly correlated with axis 1 (Fig. 3; $\mathrm{P}<0,01$ ). The impact of grazing was significant on axis 2 , being the most strongly correlated variable with this axis (Fig. 3; $\mathrm{P}<0,01$ ).

\section{Discussion}

\subsection{Soil}

Nutrient availability of soils on bowé is relatively low compared to other vegetation types in this region. In comparison to other surveys, on bowé contents of nitrogen, humus and cations as well as cation exchange capacity are slightly in- creased. Although contents on humus and nitrogen are comparatively high, the effective nutrient availability for plants is still strongly limited because of drought and low pH-values. Significant differences of bowé inside and outside the national park were not found. Hence, there is no indication of human impact on soil conditions across our study sites.

\subsection{Flora}

Grass savannas with dominance of Loudetia togoensis and most of the species encountered in this study are also described by several authors on bowé in Côte d'Ivoire (POILECOT 1995), Mali (Nasi 1994), Benin (Sieglstetter 2002) and several regions of Burkina Faso (SCHMIDT 2006; MÜLLER 2007; KroHmer 2004; Hahn-Hadjali 1998). The species composition on bowé in different climatic regions of West Africa seems to be very similar due to the extreme soil and water conditions but differs very much from the surroun- 


\begin{tabular}{|l|c|c|}
\hline & Axis 1 & Axis 2 \\
\hline Eigenvalues & 0,144 & 0,086 \\
\hline Percentage & 8,191 & 4,874 \\
\hline Cum. Percentage & 8,191 & 13,065 \\
\hline Cum.Constr.Percentage & 49,261 & 78,574 \\
\hline Spec.-env. correlations & 0,690 & 0,627 \\
\hline
\end{tabular}

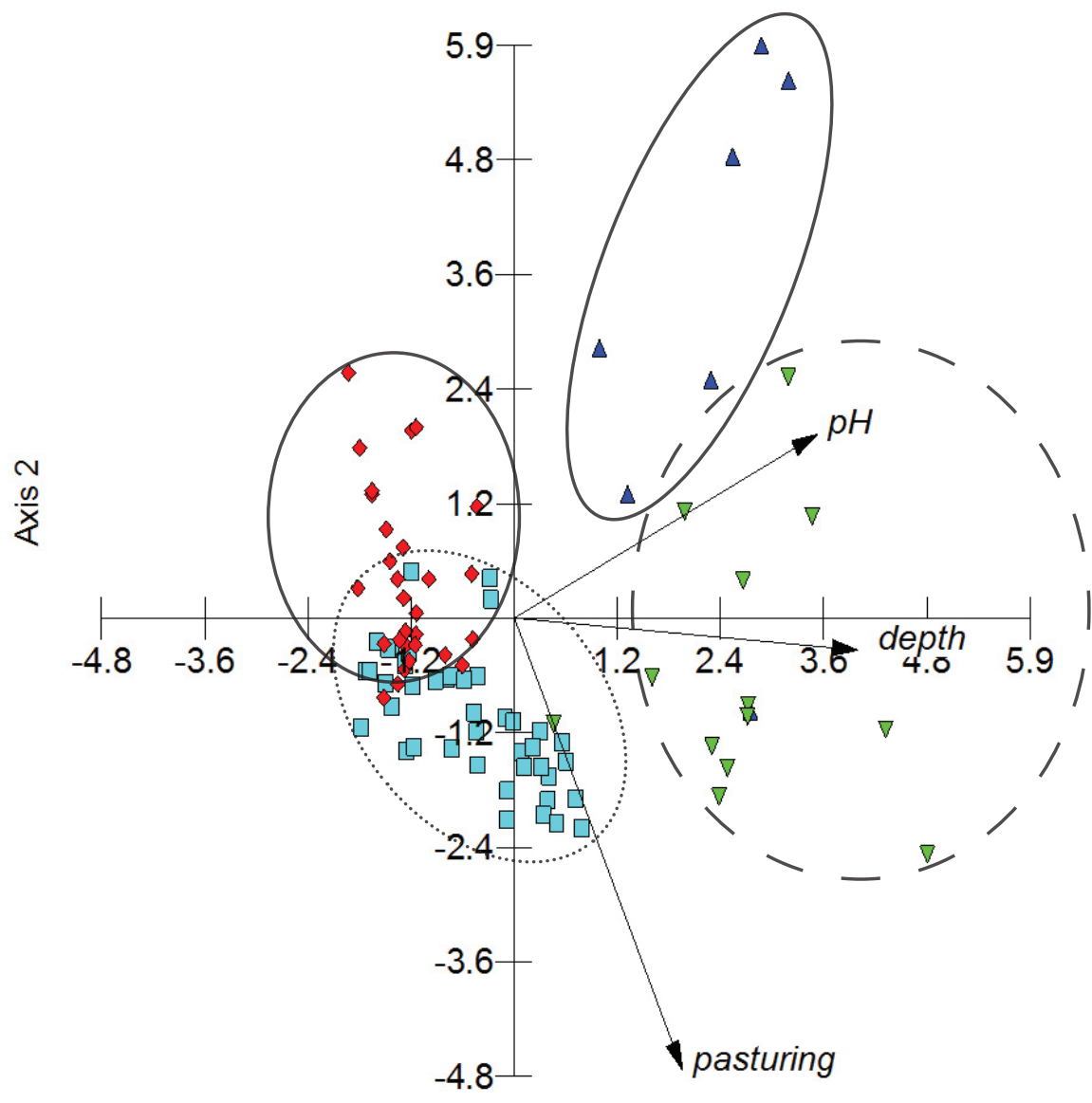

$\Delta$ protected

savannas

pastured bowé

Axis 1

Vector scalina: 5.18

Fig. 3. Ordination diagram of the CCA. The symbols represent location of the plots within the bowé or in adjacent savannas and within or outside of the W National Park. / Diagramme d'ordination CCA. Les symboles représentent la location des sites sur bowé ou savanes et dans le parc ou dehors. / Ordinationsdiagramm der CCA. Die Symbole stehen für die Lage der Aufnahmeflächen in Bowé oder angrenzenden Savannen und innerhalb oder außerhalb des W-Nationalparks.

dings. The bowé form natural grass savanna islands in savanna woodlands, which are typical for the Sudanian zone of West Africa.

Regarding species richness, the bowé are relatively poor in species compared to other vegetation types in this region (KROHMER 2004; HAHN 1996), but, due to their extreme edaphic and hydrological conditions they contain a specific flora distinct from their surroundings. This includes the dominant family Poaceae, with dominance of Loudetia togoensis or Loudetiopsis kerstingii as opposed to the typical Sudanian savannas where Andropogoneae dominate the herb layer (SCHMidT et al. 2011). Many of the bowal species do only rarely occur in other habitats. Some of them were also found on plateaus of the sandstone mountains of Gobnangou in SE Burkina Faso (KÜPPERS 1996) and inselbergs in West Africa (PoREMBSKI \& WATVE 2005) that exhibit similarly harsh edaphic conditions.

\subsection{Human impact}

In agreement with the studies of HAHN-HADJALI et al. (2006), more species were found in the pastured areas due to the occurrence of pioneers, ruderal and unpalatable plants. The increase of plants resistant to grazing as well as the decline of species sensitive to grazing outside the National Park could also be ascertained with the CCA where a significant change of floristic composition became obvious along the second axis.

\section{Conclusions}

For the first time an inventory of the flora on bowé within the W National Park and its adjacent areas has been accomplished. The examined lateritic crusts exhibit shallow acidic soils combined with impermeable compact crusts beneath. Plants are exposed to nitrogen shortage, seasonal water log- 
ging and long dry periods and exhibit functional traits in adaptation to these conditions. Most of them are therophytes and either pursue C4-photosynthesis (Poaceae, Cyperaceae, Caryophyllales) or establish symbiosis with rhizobia (Leguminosae). Therefore, the flora of lateritic crusts contains a distinct set of species that is differing from the surrounding savanna woodlands.

\section{Acknowledgements}

The investigations were part of the BMBF-funded BIOTA-Project (project code 01LC0617D1), the final compilation of the manuscript took place in the UNDESERT project (grant no. 243906) funded by the European Commission's 7th Framework program. Several samples of unknown species were determined by $\mathrm{Dr}$ Amadé Ouédraogo (University of Ouagadougou, Burkina Faso) Soil samples were analysed at the laboratory of the soil science working group, University of Frankfurt.

\section{References}

A.S.E.C.N.A. (1993): Tableaux climatologiques pour la période 1960 - 1993. Service météorologique, Ouagadougou.

AtAhOLO M (2001): Pflanzensoziologische Untersuchungen der Segetalvegetation in der Sudanzone Westafrikas. - PhD thesis, Goethe University, Frankfurt am Main. 195 p.

BOFFA JM (1999): Agroforestry parklands in sub-Saharan Africa. - FAO Conservation Guides 34, FAO, Rome.

Brown ET, Bourlès DL, Colin F, SAnfo Z, Raisbeck GM, \& YIOU F (1994): The development of iron crust lateritic systems in Burkina Faso, West Africa examined with in-situ-produced cosmogenic nuclides. - Earth Planet. Sci. Lett. 124: 19-33.

Dewis J \& Freitas F (1970): Physical and Chemical Methods of Soil and Water Analysis. - FAO Soils Bulletin 10, Rome. 275 p.

FAO-ISRIC-ISSS (1998): World Reference Base for Soil Resources. FAO, Rom.

GuINKo S (1984): Végétation de la Haute Volta. Ph.D Thesis, University of Bordeaux III. 318 p.

HAHN K (1996): Die Pflanzengesellschaften der Savannen im Südosten Burkina Fasos (Westafrika). Ihre Beeinflussung durch den Menschen und die naturräumlichen Gegebenheiten. - PhD thesis, Goethe University, Frankfurt am Main. 208 p.

HAHN-HADJALI K (1998): Les groupements végétaux des savanes du Sud - Est du Burkina Faso (l'Afrique de l'Ouest). - Etudes flore vég. Burkina Faso 3: 3-79.

Hahn-Hadjali K, Schmidt M \& Thiombiano A (2006): Phytodiversity dynamics in pastured and protected West African savannas. - In GHAZANFAR SA \& BEENTJE HJ (eds.): Taxonomy and ecology of African Plants: their conservation and sustainable use - Proc. 17th AETFAT Congress Addis Abeba 21.-26.09.2003, 351-359, Royal Botanic Gardens, Kew.

IUCN \& UNEP-WCMC (2010): The World Database on Protected Areas (WDPA). Cambridge, UK. [accessed 24 Jan 2012].

Janssen T, Schmidt M, Dressler S, Hahn K, Hien M, Konaté S, LykKe A, Mahamane A, Sambou B, Sinsin B, Thiombiano A, WitTig R, \& ZizKa G (2011): Addressing data property rights concerns and providing incentives for colla- borative data pooling: the West African Vegetation Database approach. - J. Veg. Sci. 22: 614-620.

KöHN M (1928): Bemerkungen zur mechanischen Bodenanalyse III: Ein neuer Pipettapparat. - Z. Pflanzenernähr. Düng. Bodenkd. 11: 50-54.

König K, Runge J, Schmidt M, Hahn-Hadjali K, AgbaNI P, AgONyissa D \& WiecKhorst A (2007): The impact of land use on species distribution changes in North Benin. Palaeoecol. Africa 28: 199-226.

KROHMER J (2004): Umweltwahrnehmung und -klassifikation bei Fulbegruppen in verschiedenen Naturräumen Burkina Fasos und Benins. Ph.D Thesis, Goethe University, Frankfurt am Main. 302 p.

KÜPPERS K (1996): Die Vegetation der Chaîne de Gobnangou. Ph.D Thesis, Goethe University, Frankfurt am Main. $210 \mathrm{p}$.

LAMARQue F (2004): Les Grands Mammifères du Complexe WAP. Consortium ECOPAS, CIRAD, Montpellier. 40 p.

Lebrun J-P, Toutain B, Gaston A, \& Boudet G (1991): Catalogue des plantes vasculaires du Burkina Faso. Etudes et synthèses de l'IEMVT 40. IEMVT, Maisons-Alfort. 346 p.

Mbayngone E, Schmidt M, Hahn-Hadjali K, Thiombiano A \& GuINKo S (2008): Magnoliophyta of the partial faunal reserve of Pama, Burkina Faso, Check List 4: 251-266.

Mehlich A (1948): Soil Determination of cation- and anionexchange properties of soils. Soil Sci. 66: 429-445

MÜLLER JV (2007): Herbaceous vegetation of seasonally wet habitats on inselbergs and lateritic crusts in West and Central Africa. - Folia Geobot. 42: 29-61.

NASI R (1994): La Végétation du centre régional d'endemisme soudanien au Mali. Etude de la forêt des Monts Mandingues et essaye de synthèse. PhD Thesis, University of Paris XI. 175 p. + ann.

Poilecot P (1995): Les Poaceae de Cote-d'Ivoire. Boissiera 50. Conservatoire et Jardin Botaniques de la Ville de Genève. $766 \mathrm{p}$.

PorembSKi S \& WATVE A (2005): Remarks on the species composition of ephemeral flush communities on paleotropical rock outcrops. - Phytocoenologia 35: 389-401.

RABEIL T (2003): Distribution potentielle des grands mammifères dans le Parc du W au Niger. PhD Thesis, University of Paris VII. 463 p.

SANKARAN M, RATNAM J \& HANAN N (2008): Woody cover in African savannas: the role of resources, fire and herbivory. - Glob. Ecol. Biogeogr. 17: 236-245.

SCHMidT M (2006): Pflanzenvielfalt in Burkina Faso: Analyse, Modellierung und Dokumentation. PhD Thesis, Goethe University, Frankfurt am Main. 188 p.

Schmidt M, Janssen T, Dressler S, Hahn K, Hien M, Konaté S, Lykke AM, Mahamane A, Sambou B, Sinsin B, Thiombiano A, Wittig R \& ZizKa G (2012): The West African Vegetation Database. Biodivers. Ecol. 4: 105-110.

Schmidt M, Thiombiano A, Zizka A, König K, Brunken U \& ZizKA G. (2011) Patterns of plant functional traits in the biogeography of West African grasses (Poaceae). - Afr. J. Ecol. 49: 490-500.

Sieglstetter R (2002): Vegetationsökologische und soziokulturelle Untersuchungen zur Savannenvegetation der Südsudanzone Westafrikas und ihrer Nutzung und Wahr- 
nehmung durch die ländliche Bevölkerung am Beispiel der Region Atakora im Nordwesten Benins. PhD Thesis, Goethe University, Frankfurt am Main. Band 1. 135 p.

Simioni G, LE Roux X, Gignoux J, \& Walcroft AS (2004): Leaf gas exchange characteristics and water- and nitrogen-use efficiencies of dominant grass and tree species in a West African savanna. - Plant Ecol. 173: 233-246.

TER BRAAK CJF (1988): CANOCO - an extension of DECORANA to analyze species-environment relationships. Vegetatio 75: 159-160.

Thiollay JM (2006): Large bird declines with increasing human pressure in savanna woodlands (Burkina Faso). - Biodivers. Conserv. 15: 2085-2108.

\section{AdDRESSES OF THE AUTHORS}

Alexandra Zwarg ${ }^{1,2}$, Marco Schmidt ${ }^{1,2,3}$, Thomas Janßen $^{1,4}$, Karen Hahn ${ }^{2,3}$, Georg Zizka ${ }^{1,2,3}$

${ }^{(1)}$ Research Institute Senckenberg, Dept. of Botany and molecular Evolution, Senckenberganlage 25, 60325 Frankfurt am Main

(2) Goethe University, Institute of Ecology, Evolution and Diversity, Max-von-Laue-Str. 13, 60438 Frankfurt am Main

${ }^{(3)}$ Biodiversity and Climate Research Centre (BiKF), Senckenberganlage 25, 60325 Frankfurt am Main

(4) Humboldt-Universität zu Berlin, Institute of Biology, Späth-Arboretum, Unter den Linden 6, 10099 Berlin 